\title{
Lymphadenectomy in Locally Advanced Gastric Cancer
}

\author{
Maria Sousa, Hugo Palma Rios, Teresa Carneiro, Dina Luís, António Gomes
}

Esophagogastric Unit - General Surgery Department, Hospital de Braga EPE, Portugal

\section{ABSTRACT}

Nowadays gastric cancer is still considered a fatal disease, in spite of the declined incidence and mortality due to the advances in treatment, postoperative care, and multimodality therapy. It was made a systematic research of literature published regarding gastric cancer and lymphadenectomy in locally advanced ones. Surgical resection with appropriate lymphadenectomy is still the best chance for long-term survival for patients with gastric cancer. Lymph node status is a major determinant of disease recurrence after patients undergo curative surgery. When performed in centers with experience, in a least invasive way, D2 lymphadenectomy is the procedure of choice in the treatment of locally advanced gastric cancer, guaranteeing the best survival rate.

Key words: lymphadenectomy, gastric cancer, perioperative chemotherapy

\section{INTRODUCTION}

The incidence and mortality from gastric cancer has declined dramatically since the 1930s worldwide, however, it remains a lethal disease (1). Nevertheless, Portugal has the highest rates among Western European countries (2), and gastric cancer ranks fifth in incidence and mortality when both sexes are considered (3).

Owing to the advances in surgical treatment, postoperative care, and multimodality therapy, prognosis of the gastric cancer has improved modestly over the last two decades (4). The high mortality rate is due advanced disease at presentation. Mostly of the early lesions are asymptomatic and in a few cases, are detected outside the screening program (5). Furthermore, there was a changing in the distribution of cancers from the body and antrum to the proximal stomach during the past 20 years. This lesions are biologically more aggressive and have a worse prognosis than distal gastric cancers (6). Some specific risk factors for cancer of the stomach or gastroesophageal junction include a genetic predisposition (eg, hereditary nonpolyposis colorectal cancer, familial polyposis coli, and hereditary diffuse gastric cancer), dietary factors, acid hyposecretory conditions, infection with Helicobacter pylori, and Barrett's esophagus (7).

Surgical resection still is the best chance for long-term survival for patients with localized gastric cancer, particularly in combination with
Corresponding author: Hugo Palma Rios, MD Esophagogastric Unit - General Surgery Department Hospital de Braga EPE, Portugal E-mail: hugojoaorios@gmail.com
Received: 13.11.2019

Accepted: 12.12.2019 
adjuvant or perioperative chemotherapy or chemoradiotherapy (if indicated). In some cases of advanced disease, surgery might be necessary for staging and palliation.

\section{Gastric cancer - staging evaluation}

In order to obtain the optimal therapy, it's crucial an accurate staging of the extent of disease. That includes physical examination, CT (chest, abdomen and pelvis), EUS (endoscopic ultrasound) and eventually staging laparoscopy.

EUS may provide more accurate staging evaluation of the tumor (T) and nodal (N) stage than CT and also allows for preoperative biopsies. The staging laparoscopy has the advantage of directly visualizing the liver surface, peritoneum, and local lymph nodes, permitting biopsy of any suspicious lesions and identify radiographically occult metastatic disease and assess the resectability of primary tumor, avoiding unnecessary exploratory laparotomy. At laparoscopy, peritoneal metastases are documented in $20-30 \%$ of Western patients with gastric cancer with a negative $\mathrm{CT}$, who would have been otherwise considered potentially resectable. In addition, peritoneal cytology should be obtained during staging laparoscopy. Although, it is a controversial theme, some experts indicate staging laparoscopy for patients with EUS stage T3/4 disease (8). In the absence of secondary lesions on staging exams, a positive peritoneal cytology carries a high risk of progression to carcinomatosis (9).

The most frequently used staging system is TNM (tumor, node, metastasis). In the 2017 revision of TNM classification, tumors involving the esophagogastric junction (EGJ) with the tumor epicenter no more than $2 \mathrm{~cm}$ into the proximal stomach are staged as esophageal cancers (Siewert I/II). EGJ tumors with their epicenter located more than $2 \mathrm{~cm}$ into the proximal stomach are staged as stomach cancers (Siewert III), even if they involve the EGJ, as are all cardia cancers not involving the EGJ (10).

The preoperative evaluation allows the assessment of clinical stage, which is important in guiding decisions about preoperative or perioperative therapy.

\section{Surgical treatment for localized disease}

The complete surgical removal with adequate lymphadenectomy is the best chance for long-term survival in gastric cancer treatment (11). The presence and number of involved lymph nodes is one of the most important prognostic indicators.
There are some widely accepted criteria of unresectability for gastric cancer: the presence of distant metastases, invasion of a major vascular structure, such as the aorta, or disease encasement or occlusion of the hepatic artery or celiac axis/proximal splenic artery.

The lymphatics around the stomach are rich, and the presence of locoregional lymph node metastases that are located geographically distant from the tumor should not necessarily be considered an indicator of unresectability. Involvement of other intraabdominal nodal groups (ie, pancreatoduodenal, retropancreatic, peripancreatic, superior mesenteric, middle colic, paraaortic, and retroperitoneal) is classified as distant metastases (10).

However, patients who have bulky adenopathy fixed to the pancreatic head that might indicate the need for a Whipple procedure are at a high risk for occult metastatic disease. In these cases, staging laparoscopy and/or upfront chemotherapy should be strongly considered rather than surgery initially. Lymph nodes behind or inferior to the pancreas, aorto-caval region, into the mediastinum, or in the porta hepatis are typically considered outside of the surgical field and thus evidence of unresectability. These nodes would fall into areas that would be defined as third or fourth echelon nodes in the Japanese nomenclature.

\section{Lymph node dissection}

The number of examined nodes influences the accuracy of staging, and influences survival (12). The location of positive nodes did not significantly affect median survival, while the number of positive lymph nodes had a profound influence on survival (13). The AJCC staging guidelines recommend that at least 16 regional nodes be assessed pathologically but that removal/evaluation of more nodes (30 or more) is desirable (10).

In highly differentiated centers in Japan and Korea, extensive lymphadenectomy is performed, which can contribute to the best outcomes compared to Western countries (14). However, even these surgeons are increasingly moving away from the most aggressive dissections.

The Japanese Research Society for Gastric Cancer categorizes the draining lymph node basins of the stomach into 16 stations, including 6 perigastric stations and 10 regional stations close to major vessels, pancreas and aorta (15).

The extent of lymphadenectomy is indicated by the designation $\mathrm{D}$ :

- D1 dissection includes the perigastric lymph 
nodes (stations 1 to 7 ). In the Japanese literature, a D1+ lymphadenectomy refers to a D1 lymphadenectomy plus stages $8 \mathrm{a}, 9$, and $11 \mathrm{p}$.

- D2 dissection includes the lymph nodes in common hepatic, left gastric, celiac, splenic arteries and the splenic hilum (stations 1 to 12a).

- D3 dissection includes nodes within the porta hepatis and adjacent to the aorta (stations 1-16). From the perspective of Western surgeons, lymphadenectomy of these lymph nodes is not recommended because they consider that they are distant metastasis.

- Most Western surgeons classify disease in these regions as distant metastasis and do not recommend removing nodes in these areas.

\section{Extent of lymphadenectomy - current news}

The type of gastrectomy will dictate how far will be the lymphadenectomy regardless of tumor location according to recent Japanese gastric cancer treatment guidelines. However, lymphatic flow from lower- third stomach mainly drain to supra- and infra-pyloric nodes, as well as to partially lesser curvature to the left gastric artery. Although, the frequencies of lymph nodes (LN) metastases at No. 1 and No. 4sb stations were rare in patients with cancer of lower-third stomach. Survival benefit of dissection of these two LN was presumed to be extremely low, suggesting that lymphadenectomy along the upper-third-stomach could be omitted in cancer of lower-third stomach patients when undergoing distal gastrectomy (16).

There is no consensus over the type of resection and lymphadenectomy extension that could be a standard of care in junctional cancer. In 2012-2013, it was constructed an algorithm based on surveillance, showing the tentative standard in the extent of lymphadenectomy based on the tumor location, histology and T-categories by the Japanese Gastric Cancer Association and Japan Esophageal Society.

Since the pre- and intraoperative diagnoses of lymph node metastases remain unreliable, a D2 lymphadenectomy should be performed regardless of nodal status. A D1 lymphadenectomy is recommended for T1a tumors that do not have the criteria for endoscopic resection, and for cT1bNO tumors that are histologically of differentiated type and $1.5 \mathrm{~cm}$ or smaller in diameter. In case of cT1N0 tumors other than the above, D1+ lymphadenectomy is indicated. A D2 lymphadenectomy should be applied for potentially curable T2-T4 tumors as well as cT1N+tumors. The role of splenectomy for complete resection of Nos. 10 and
11 nodes had long been an issue of controversy, and the results of a randomized trial (JCOG 0110) are expected.

Most highly differentiated centers are performing a D2 in order to the positive impact on disease-specific survival. The NCCN (17) guidelines recommend that gastric cancer resection involve the regional lymphatics, including perigastric nodes, those along the left gastric artery, common hepatic artery, celiac artery, splenic hilum, and splenic artery (D2 lymph nodes), with the aim of examining 15 or more lymph nodes.

There is no evidence that a D3 (paraaortic) lymphadenectomy confers a survival benefit over a D2 dissection, and it is associated with greater perioperative mortality and lack of a survival benefit in most large randomized trials. Furthermore, routine splenectomy or pancreatectomy during gastric resection should be discouraged as it increases morbidity and mortality without improving survival. It should only be considered if there is evidence of direct tumor extension to the pancreas or spleen.

A systematic review and meta-analysis identified 28 articles reporting the relationship between hospital and surgeon factors with procedure-related morbidity, procedure- related mortality, and five-year survival for gastric cancer surgery (18). Higher hospital volume was associated with lower, unadjusted procedure- related mortality. Surgeon volume, level of training, hospital volume, and specialization were also associated with procedure-related morbidity, procedure- related mortality, and five-year survival, but the effect was not consistent across all studies.

Several trials published in the literature originating from Western and Eastern reference centers have not shown an overall survival benefit between D2 and D1 lymphadenectomy $(19,20)$ or between D3 and D2 lymphadenectomy (21). Two of three separate metaanalyses comparing D1 with D2 node dissection have concluded that there is no survival benefit to D2 lymphadenectomy, although the most recent and inclusive analysis of five randomized controlled trials did find a modest advantage for D2 lymphadenectomy in terms of disease-specific survival, which was counterbalanced by a twofold higher rate of postoperative mortality (22). Both of the meta-analyses concluded that there is no significant survival benefit from a more extended lymphadenectomy than D2 $(22,23)$.

When we compare D1 with D2, most of the multiple randomized trials have been unsuccessful to demonstrate a survival benefit for D2 versus D1. However, there were some concerns about the Dutch and the Medical Research Council [MRC] trials because it was considered underpowered to show a survival 
advantage to D2 lymphadenectomy (15).

The MRC randomly assigned 400 patients in two arms: D1 or D2 lymphadenectomy (24). The results shown a significantly higher morbidity (46 versus 28 percent) and mortality ( 13 versus $6 \%$ ) in the D2 group. This difference is related with an extensive lymphadenectomy associated with splenectomy and distal pancreatectomy. Long-term, survival rates were comparable despite the increased operative mortality (five-year survival rates 33 versus $35 \%$ ) (25).

In the case of the Dutch trial, 711 patients were enrolled. The surgical team was trained by a Japanese surgeon with extensive experience in lymphadenectomy. However, the number of nodal stations removed was very inconsistent, which made it difficult to interpret the results (25). Morbidity (43 versus $25 \%$ ) and mortality (10 versus $4 \%$ ) were higher in the D2 group. No survival advantage in the D2 group was observed in short and long term follow-up. However, a significantly lower risk of recurrence was noted (26).

At the base of the Dutch trial's organization was the idea that an extensive lymphadenectomy would increase survival from 20 to $32 \%$. In addition, the percentage of patients with early gastric cancer was higher than expected (around 40\%).

The results of this study were reviewed after 15 years. (27). There are still no differences in overall survival ( 22 versus $28 \%$ in the D1 and D2 arms, respectively, $p=0.34)$. However, the gastric cancer-related death rate is significantly higher in the D1 arm (48 versus $37 \%$ ). These results suggest that if D2 lymphadenectomy is performed with low morbidity and mortality, it may have an impact on survival.

In a recent review by Cochrane (20), there was no significant advantage for D2 lymphadenectomy for overall survival or disease-free survival, there was a significant difference in disease-specific survival, favoring D2 lymphadenectomy (HR 0.81, 95\% Cl 0.710.92 ). The quality of the evidence was judged as moderate. However, D2 lymphadenectomy was also associated with a high postoperative mortality rate.

In conclusion, both NCCN (17) and ESMO (28) recommend D2 lymphadenectomy for patients with potentially curable gastric cancer.

Concerning D3 dissection, the Japan Clinical Oncology Group (JCOG) study 9501 randomly assigned 523 patients to $D 2$ or $D 3$. The morbidity in the $D 3$ group was significantly higher (28.1 versus $20.9 \%$ ). However, there were no differences in significant complications (anastomotic leak, pancreatic fistula, abdominal abscess, pneumonia). Mortality was very low $(0.8 \%)$ in both groups (29). Five-year recurrence-free (approxi- mately $63 \%$ in both groups) and overall survival (70 versus $69 \%$ ) were not improved with D3 lymphadenectomy (21).

One of the confounding issues with the JCOG trial is that in subgroup analysis, patients with node negative disease fared significantly better with the more aggressive D3 operation than with D2 lymphadenectomy. Conversely, patients who were node- positive fared significantly better with a D2 lymphadenectomy than with more aggressive D3 surgery (21). The reasons for these counterintuitive results are unclear. Data from the JCOG trial as well as those from other groups (29). suggest that a D2 dissection can be performed safely with a perioperative mortality rate that is under 2 percent. The 2015 Cochrane analysis of the JCOG trial and two other smaller randomized trials concluded that lymphadenectomy of the paraaortic nodes was not associated with longer survival (22).

Furthermore, para-aortic metastases from gastric cancer are classified as M1, and surgery with curative intent is not indicated. However, neoadjuvant chemotherapy followed by $\mathrm{D} 2+$ is a promising option. A phase II trial analyzed the systemic para-aortic lymph node dissection, reporting a $53 \%$ of 5 -year survival. So, this treatment strategy could be an option for institutions with sufficient expertise on this dissection (30). Conversely, these retrospective studies are known to include patients who eventually underwent surgery after responding to the chemotherapy and may at least partially reflect the benefit of the multidisciplinary approach (31).

Moreover, a recent randomized trial from Taiwan demonstrated a statistically significant survival advantage associated with D3 versus D1 dissection (32) and the most recent update of the Dutch D1/D2 trial showed that D2 dissections to be associated with improved disease-specific survival over D1 dissections (lower disease-related death rate, $37 \%$ vs $48 \%$, respectively) (27).

The accurate staging of patients with gastric cancer, according to AJCC Cancer staging manual 8th edition, demands the evaluation of at least 16 lymph nodes (33). The nodal staging is then based on the number of positive nodes, with N1 (1-2 positive nodes), N2 (3-6 positive nodes), N3a (7-15 positive nodes), and N3b (>16 positive nodes) categories. Retrospective studies have shown a correlation between improved patient survival and larger numbers of lymph nodes examined in the specimen (34). There is indirect and recent direct evidence, however, that a D2 lymph node dissection may result in lower rates of locoregional recurrence, and this may translate into a survival benefit for gastric 
adenocarcinoma patients (27).

Lymph node status is a major determinant of disease recurrence (35). However, even among patients without LN metastasis (NO), recurrence may occur. In gastric carcinoma, the significance of micrometastases in lymph nodes remains controversial. Most published reports suffer from the problems of small sample size and selection bias (35).

In some of these cases, occult tumor cells (OTC) are thought to play an important role and seems to occur with a variable prevalence. The majority of the retrieved studies (75\%) evaluating the predictive role of OTC conclude that its presence is associated with a worse prognosis (36). Controversally, the presence of immunohistochemically detected micrometastases in the regional lymph nodes did not affect the survival of Japanese patients with PT2NOMO gastric carcinoma who had undergone D2 lymph node dissection (35).

For Siewert type II adenocarcinoma, the optimal surgical approach and extent of lymph nodes dissection remain controversial. The immunohistochemistre has been reported to be available for identifying lymph node micrometastasis and as a positive prognostic factor, thorough lower mediastinal lymphadenectomy in an invasive approach should be considered when necessary (37).

\section{CONCLUSION}

Even in the age of neoadjuvant treatment, radical surgery with adequate lymphadenectomy is the primary treatment of gastric cancer. This type of surgery should be performed in centers with high experience, in the least invasive way possible and with the lowest possible morbidity. As described in the literature, D2 lymphadenectomy is the procedure of choice in the treatment of locally advanced gastric cancer, ensuring the best survival rate.

\section{Disclosure}

No commercial or financial conflicts of interest related to this article.

\section{REFERENCES}

1. Zhu AL, Sonnenberg A. Is gastric cancer again rising? J Clin Gastroenterol. 2012;46(9):804-6.

2. Morais S, Ferro A, Bastos A, Castro C, Lunet N, Peleteiro B. Trends in gastric cancer mortality and in the prevalence of Helicobacter pylori infection in Portugal. Eur J Cancer Prev. 2016;25(4):275-81.

3. Ferlay J, Soerjomataram I, Ervik M, Dikshit R, Eser S, Mathers C, et al. Cancer incidence and mortality worldwide: sources, methods and major patterns in GLOBOCAN 2012. Int J Cancer. 2015;136(5):
E359-86.

4. Hyung W, Kim S, Choi WH, Cheong JH, Choi SH, Kim CB, et al. Changes in treatment outcomes of gastric cancer surgery over 45 years at a single institution. Yonsei Med J. 2008;49(3):409-415.

5. Yoshida S, Saito D. Gastric premalignancy and cancer screening in high-risk patients. Am J Gastroenterol. 1996;91(5):839-43.

6. Wanebo HJ, Kennedy BJ, Chmiel J, Steele G Jr, Winchester D, Osteen R. Cancer of the stomach. A patient care study by the American College of Surgeons. Ann Surg. 1993;218(5):583-92.

7. Sue-Ling HM, Johnston D, Martin IG, Dixon MF, Lansdown MR, McMahon MJ, et al. Gastric cancer: a curable disease in Britain. BMJ 1993;307(6904):591-6.

8. Power DG, Schattner MA, Gerdes H, Brenner B, Markowitz AJ, Capanu M, et al. Endoscopic ultrasound can improve the selection for laparoscopy in patients with localized gastric cancer. J Am Coll Surg 2009;208(2):173-8.

9. De Andrade JP, Mezhir JJ. The critical role of peritoneal cytology in the staging of gastric cancer: an evidence-based review. J Surg Oncol. 2014;110(3):291-7.

10. Ajani JA, In H, Sano T, et al. Stomach. In: AJCC Cancer Staging Manual, 8th, Chicago: Amin MB. (Ed), AJC C: 2017. p.203.

11. Fugazzola P, Ansaloni L, Sartelli M, Catena F, Cicuttin E, Leandro G, et al. Advanced gastric cancer: the value of surgery. Acta Biomed. 2018;89 (Suppl 8):110-116.

12. Woo $\mathrm{Y}$, Goldner B, Ituarte $\mathrm{P}$, Lee $\mathrm{B}$, Melstrom L, Son $\mathrm{T}$, et al. Lymphadenectomy with Optimum of 29 Lymph Nodes Retrieved Associated with Improved Survival in Advanced Gastric Cancer: A 25,000-Patient International Database Study. J Am Coll Surg. 2017; 224(4):546-555

13. Karpeh MS, Leon L, Klimstra D, Brennan MF. Lymph node staging in gastric cancer: is location more important than Number? An analysis of 1,038 patients. Ann Surg 2000; 232:362(3):362-71.

14. Noguchi $Y$, Yoshikawa T, Tsuburaya A, Motohashi H, Karpeh MS, Brennan MF. Is gastric carcinoma different between Japan and the United States? Cancer 2000; 89(11):2237-46.

15. Eleftherios AM, George A. Surgical considerations in the management of gastric adenocarcinoma. Surg Clin N Am. 2017;97 (2):295-316.

16. Saito R, Kawaguchi $Y$, Akaike H, Shiraishi K, Maruyama S, Shimizu $\mathrm{H}$, et al. Prognostic significance of lymph node dissection along the upper-third-stomach in patients with lower-third Gastric Cancer. Anticancer Res. 2019;39(3):1485-1489.

17. National Comprehensive Cancer Network (NCCN) Clinical Practice Guidelines in Oncology. https://www.nccn.org/professionals/ physiciangls/pdf/aml.pdf

18. Mahar AL, McLeod RS, Kiss A, Paszat L, Coburn NG. A systematic review of the effect of institution and surgeon factors on surgical outcomes for gastric cancer. J Am Coll Surg. 2012;214(5): 860-8.e12.

19. El-Sedfy A, Dixon M, Seevaratnam R, Bocicariu A, Cardoso R, Mahar A, et al. Personalized surgery for gastric adenocarcinoma: a meta-analysis of D1 versus D2 lymphadenectomy. Ann Surg Oncol. 2015;22(6):1820-7.

20. Degiuli M, Sasako M, Ponti A, Vendrame A, Tomatis M, Mazza C, et al. Randomized clinical trial comparing survival after D1 or D2 gastrectomy for gastric cancer. Br J Surg. 2014;101(2):23-31.

21. Sasako M, Sano T, Yamamoto S, Kurokawa Y, Nashimoto A, Kurita $A$, et al. D2 lymphadenectomy alone or with para-aortic nodal dissection for gastric cancer. N Engl J Med 2008;359(5):453-62.

22. Mocellin S, McCulloch P, Kazi H, Gama-Rodrigues JJ, Yuan Y, Nitti D. Extent of lymph node dissection for adenocarcinoma of the stomach. Cochrane Database Syst Rev. 2015;(8):CD001964.

23. Jiang L, Yang KH, Guan QL, Zhao P, Chen Y, Tian J-H. Survival and recurrence free benefits with different lymphadenectomy for resectable gastric cancer: a meta-analysis. J Surg Oncol 2013; 107(8):807-14.

24. Cuschieri A, Fayers P, Fielding J, Craven J, Bancewicz J, Joypaul V, et al. Postoperative morbidity and mortality after D1 and D2 resections for gastric cancer: preliminary results of the MRC randomised controlled surgical trial. The Surgical Cooperative Group. Lancet 1996;347(9007):995-9. 
25. Hartgrink HH, van de Velde CJ, Putter H, Bonenkamp JJ, Klein Kranenbarg E, Songun I, et al. Extended lymph node dissection for gastric cancer: who may benefit? Final results of the randomized Dutch gastric cancer group trial. J Clin Oncol 2004; 22(11):2069-77.

26. Bonenkamp JJ, Hermans J, Sasako M, van de Velde C J, Welvaart K, Songun I, et al. Extended lymph-node dissection for gastric cancer. N Engl J Med 1999;340(12):908-14.

27. Songun I, Putter H, Kranenbarg EM, Sasako M, van de Velde CJH. Surgical treatment of gastric cancer: 15- year follow-up results of the randomised nationwide Dutch D1D2 trial. Lancet Oncol 2010;11(5):439-49.

28. Gastric cancer: European Society for Medical Oncology (ESMO) clinical practice guidelines. https://www.esmo.org/guidelines/ gastrointestinal-cancers/gastric-cancer

29. Sano T, Sasako M, Yamamoto S, Nashimoto A, Kurita A, Hiratsuka $\mathrm{M}$, et al. Gastric cancer surgery: morbidity and mortality results from a prospective randomized controlled trial comparing D2 and extended para-aortic lymphadenectomy-- Japan Clinical Oncology Group study 9501. J Clin Oncol 2004; 22(14):2767-73.

30. Tsuburaya A, Mizusawa J, Tanaka Y, Fukushima N, Nashimoto A Sasako M, et al. Neoadjuvant chemotherapy with S-1 and cisplatin followed by D2 gastrectomy with para-aortic lymph node dissection for gastric cancer with extensive lymph node metastasis. Br J Surg. 2014;101(6):653-60
31. Japanese Gastric Cancer Association. Japanese gastric cancer treatment guidelines 2014 (ver. 4). Gastric Cancer 2017;20(1):1-19.

32. Wu CW, Hsiung CA, Lo SS, Hsieh MC, Chen JH, Li A FY, et al. Nodal dissection for patients with gastric cancer: a randomised controlled trial. Lancet Oncol 2006;7(4):309-15.

33. Amin MB, Edge S, Greene F, et al. AJCC Cancer staging manual. 8th edition; 2017.

34. Gholami S, Janson L, Worhunsky DJ, Tran TB, Hart Squires M 3rd, Jin LX, et al. Number of lymph nodes removed and survival after gastric cancer resection: an analysis from the US Gastric Cancer Collaborative. J Am Coll Surg 2015;221(2):291-9.

35. Fukagawa T, Sasako M, Mann GB, Sano T, Katai H, Maruyama K, et al. Immunohistochemically detected micrometastases of the lymph nodes in patients with gastric carcinoma. Cancer. 2001;92(4): $753-60$.

36. Tavares A, Monteiro-Soares M, Viveiros F, Maciel Barbosa J, Dinis-Ribeiro M. Occult tumor cells in lymph nodes of patients with gastric Cancer: a systematic review on their prevalence and predictive role. Oncology. 2015;89(5):245-54.

37. Zheng B, Ni CH, Chen H, Wu WD, Guo ZH, Zhu Y, et al. New evidence guiding extent of lymphadenectomy for esophagogastric junction tumor: Application of Ber-Ep4 Joint with CD44v6 staining on the detection of lower mediastinal lymph node micrometastasis and survival analysis. Medicine (Baltimore). 2017;96(14):e6533. 